\title{
Emerging targeted combinations in the management of breast cancer
}

\author{
This article was published in the following Dove Press journal: \\ Breast Cancer:Targets and Therapy \\ 12 August 2013 \\ Number of times this article has been viewed
}

\section{Rebecca J Lee \\ Anne C Armstrong \\ Andrew MWardley \\ Department of Medical Oncology, The Christie NHS Foundation Trust, Wilmslow Road, Manchester, UK}

Correspondence: Dr Andrew MWardley Cancer Research UK Department of Medical Oncology, The Christie, 550 Wilmslow Road, Manchester, M20 4BX, UK

$\mathrm{Tel}+44$ I6I 4463746

Fax +44 I6।4468564

Email andrew.wardley@christie.nhs.uk

\begin{abstract}
The number of targeted treatments has risen exponentially over the last few years and is an important concept in the fight against cancer. This review will concentrate on some of the main treatments targeting aberrant pathways which have been tested mainly in the Phase I/II setting. These include human epidermal growth factor receptor 2 inhibitors, drug-antibody conjugates, epidermal growth factor receptor inhibitors, vascular endothelial growth factor inhibitors, reticular activating system, mammalian target of rapamycin and multi-kinase inhibitors. Further knowledge of these pathways and the predictors of response to them will enable personalized medicine to become a reality.
\end{abstract}

Keywords: breast cancer, targeted therapy, tyrosine kinase, receptor

\section{Introduction}

With the discovery of estrogen receptors, targeted treatments became a reality. Since then, attention has turned to molecular pathways and alternative receptors as potential targets. As our knowledge of the mechanisms behind cancer cell development has improved, so too has our ability to develop therapies that can inhibit aberrant pathways. This review will examine some of the main drugs that have been investigated during the last few decades.

\section{HER-2 receptor}

The neu oncogene was first discovered in 1984 by Schechter et al. ${ }^{1}$ It is a member of the epidermal growth factor receptor family (EGFR, see Figure 1) and is encoded by the proto-oncogene "v-erb-b2 erythroblastic leukemia viral oncogene homolog 2, neuro/ glioblastoma derived oncogene homolog (avian)." The human equivalent is her-2, which is found on chromosome $17 \mathrm{q} 21.1 .^{2}$ HER2 overexpression, found in approximately $22 \%$ of breast cancers, ${ }^{3}$ is a marker for a more aggressive phenotype with increased growth rates, increased likelihood of early metastasis, and decreased overall survival (OS). ${ }^{2}$

The discovery of HER2 has enabled a variety of targeted therapies to be developed. The first of these was trastuzumab $\left(\right.$ Herceptin $\left.^{\circledR}\right)$, a monoclonal antibody which binds to the extracellular juxtamembrane protein of HER2, therefore blocking its ability to promote cellular proliferation and survival. ${ }^{4}$ Slamon et al conducted a Phase III trial in the metastatic setting which found a statistically significant improvement in overall response rate (ORR) $(50 \%$ versus $32 \%, P<0.001)$, progression free survival (PFS) (7.4 months versus 4.6 months $P<0.001)$ and, importantly, overall survival (OS) (25.1 months versus 20.3 months, $P=0.046)$ when trastuzumab was combined 


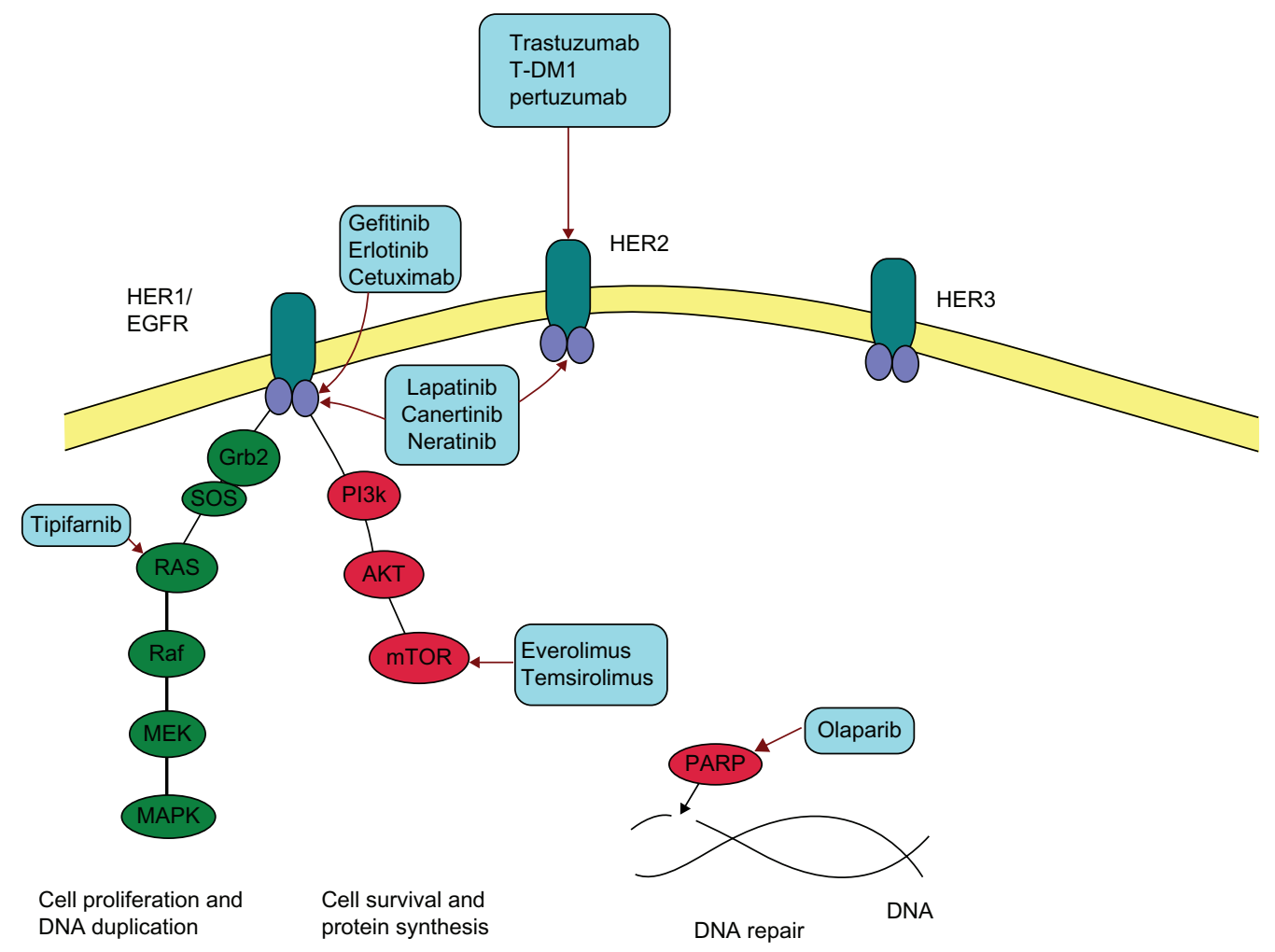

Figure I Human epidermal growth factor receptor (HER) and poly (ADP-ribose) polymerase (PARP).

Abbreviations: AKT, also known as Protein Kinase B (PKB), is a serine/threonine-specific protein kinase; Grb2, growth factor receptor-bound protein 2; HER, human epidermal growth factor receptor; MAPK, mitogen-activated protein kinases; MEK (aka MAP2K), mitogen-activated protein kinase kinase; mTOR, mammalian target of rapamycin; PARP, poly (ADP-ribose) polymerase (PARP); PI3k, phosphatidylinositol 3'-kinase; Raf, protooncogene cytoplasmic serine/threonine protein kinases; SOS, son of sevenless.

with different chemotherapy regimens. ${ }^{2}$ As a result of this study, a new standard of care for patients with metastatic HER2 positive (HER2+) breast cancer was defined.

In the adjuvant setting, a number of large Phase III studies again showed benefit for adding trastuzumab to standard chemotherapy. ${ }^{5}$ The NCCTG, N9831, and NSABP B-31 trials examined doxorubicin, cyclophosphamide, and paclitaxel with or without trastuzumab following surgery for HER2+ breast cancers. ${ }^{5}$ In the combined analysis, for a total of 3969 participants at median follow up of 2.9 years (range up to 6.4 years), the 4-year disease-free survival (DFS) rate was $85.9 \%$ in the trastuzumab arm compared with $73.1 \%$ in the non-trastuzumab arm; hazard ratio (HR) $0.49(P<0.0001$; 95\% confidence interval [CI]: 0.41-0.58). The four-year OS for the combination with trastuzumab was $92.6 \%$ versus 89.4\% without; HR 0.63 ( $P=0.0004$; 95\% CI 0.49-0.81). ${ }^{5}$

The multicenter HERA trial randomized 5102 women who had received locoregional therapy and a minimum of four courses of adjuvant or neoadjuvant chemotherapy to receive either observation alone (number $[\mathrm{n}]=1698$ ), trastuzumab for 1 year $(\mathrm{n}=1703)$ or 2 years $(\mathrm{n}=1701) .{ }^{6}$ At median follow up of 23.5 months (range 0-48 months), the 1-year trastuzumab arm had a 3-year DFS of $80.6 \%$ compared with $74.3 \%$ in the observation arm; HR 0.63 (95\%
CI $0.53-0.75 ; P<0.0001)$. The 3 -year OS was $2.7 \%$ better with 1-year trastuzumab compared with observation; HR 0.63 $(0.45-0.87 ; P=0.0051){ }^{6}$

Finally, the BCIRG 006 study compared combinations of trastuzumab with anthracycline or non-anthracycline based chemotherapy. ${ }^{7}$ The investigators randomized 3222 women with early stage breast cancer HER $2+$ following surgery to receive either docetaxel, cyclophosphamide, and doxorubicin (AC-T) or AC-T plus trastuzumab or docetaxel, carboplatin and trastuzumab (TCH). Both trastuzumab containing regimens had significantly improved PFS and OS compared with AC-T alone. There was no significant difference in OS or PFS between the two trastuzumab regimens, but there was greater congestive cardiac failure seen in the anthracycline containing regimen compared with $\mathrm{TCH}(2.0 \%$ versus $0.4 \% P<0.001) .^{7}$ Loss of mean left ventricular ejection fraction (defined as $>10 \%$ relative loss) was $18.6 \%$ in the AC-T plus trastuzumab arm versus $9.4 \%$ in the TCH arm $(P<0.001)$ and this was still present in $33 \%$ of those patients at 4 years.?

\section{Pertuzumab}

The monoclonal antibody pertuzumab (Perjeta ${ }^{\circledR}$ ) also targets HER 2 but binds to a different epitope to trastuzumab 
(subdomain II compared to subdomain IV) preventing dimerization of HER $2 .{ }^{8}$ Normally, subdomain II is responsible for dimerization of HER2 with either other HER2 receptors (homodimerization) or HER1/HER3 receptors (heterodimerization). ${ }^{8}$ The pairing of receptors results in a cascade of signaling that promotes tumor growth ${ }^{8}$ and may also affect tumor resistance to therapies. ${ }^{9}$

A Phase II study comparing two doses of pertuzumab in 78 women with HER2 negative metastatic breast cancer (MBC) whose disease had progressed through up to two lines of previous therapy resulted in limited efficacy..$^{10}$ Only two patients had a partial response (PR) with 44\% (18/41) of patients in the $420 \mathrm{mg}$ dose arm having stable disease (SD) and 38\% (14/37) of patients in the $1050 \mathrm{mg}$ arm having SD lasting $>12$ weeks. ${ }^{10}$ The authors recommended that pertuzumab should not be used as a single agent in unselected patients. ${ }^{10}$ However, as pertuzumab and trastuzumab have different mechanisms of action with pertuzumab acting as a dimerization inhibitor of HER2 compared with trastuzumab which inhibits HER2 cleavage, it was hypothesized that they would work synergistically. A Phase II study performed in 66 patients with HER2+ MBC who had previously had trastuzumab in which they received pertuzumab in combination with trastuzumab demonstrated an ORR of $24.2 \%$ with $7.6 \%$ patients experiencing complete remission (CR) and $16.7 \%$ a $\mathrm{PR}$, despite the documented resistance to single agent trastuzumab. ${ }^{11}$

These results led to the Phase III CLEOPATRA study which randomized 808 patients with previously untreated HER2+ MBC to receive placebo, trastuzumab, and docetaxel or pertuzumab, trastuzumab, and docetaxel. ${ }^{12}$ The investigators demonstrated a median PFS of 12.4 months in the control arm versus 18.5 months in the pertuzumab group (HR $0.62 ; 95 \%$ CI $0.51-0.75 ; P<0.001) .{ }^{12}$ Interim analysis at median 30 months follow up and 267 deaths (69\% of planned events for the final analysis), demonstrated a median OS of 37.6 months for the placebo arm and had not yet been reached for the pertuzumab arm. The HR was significantly in favor of pertuzumab $(\mathrm{HR}=0.66 ; 95 \% \mathrm{CI} 0.52-0.84 ; P=0.0008) .{ }^{13}$ Dual targeting of a single receptor is superior, for HER2 at least, to monotherapy.

\section{Trastuzumab-DM1}

Trastuzumab-DM1 (T-DM1) or trastuzumab-emtansine as it is alternatively known, represents an interesting development in targeted chemotherapy delivery. It is an antibodydrug conjugate containing trastuzumab, which is covalently bonded to the chemotherapy agent emtansine. ${ }^{14}$ Emtansine is derived from maytansine, which binds to microtubules, thereby preventing mitosis. It has previously been shown to have good cytotoxic activity, but its clinical utility was limited by its toxicity. It has however been possible to covalently bind emtansine to trastuzumab with the linker molecule ( $N$-maleimidomethyl) cyclohexane-1-carboxylate. ${ }^{14}$

In a Phase I trial Krop et al demonstrated a confirmed objective tumor response (OR) in 5/24 heavily pretreated patients with HER2+ metastatic breast cancer (MBC).$^{15} \mathrm{Of}$ the 15 patients treated at the maximum tolerated dose of $3.6 \mathrm{mg} / \mathrm{kg}, 73 \%$ achieved either OR or SD. ${ }^{15}$

Evidence of its activity was further validated in a Phase II trial of 112 patients with HER2+ MBC, whose disease had progressed through prior treatment with both trastuzumab and chemotherapy (median number of prior treatments was 8, range $2-19) .{ }^{16}$ ORR in this heavily pretreated population was $25.9 \%$ (95\% CI 18.4\%-34.4\%) by independent assessment with a follow up of greater than 12 months. Median PFS was 4.6 months (95\% CI 3.9-8.6 months). It was generally well tolerated with the majority of events being grade 2 or less. The commonest grade 3 adverse events (AE) were hypokalemia $(8.9 \%)$, thrombocytopenia $(8.0 \%)$, and fatigue $(4.5 \%){ }^{16}$

EMILIA, a Phase III trial comparing T-DM1 with lapatinib combined with capecitabine in 991 HER2+ patients with MBC was recently published. ${ }^{17}$ Patients had received both prior trastuzumab and a taxane. PFS was significantly better in the T-DM1 arm (9.6 months versus 6.4 months $P<0.001) .{ }^{17}$ At the second interim analysis of OS at 331 deaths, T-DM1 median OS was 30.9 months versus 25.1 months with lapatinib plus capecitabine $P<0.001$. ORR was also greater in the T-DM1 arm (43.6\%) than in the lapatinib capecitabine arm $(30.8 \%) P<0.001$. Frequently occurring toxicities were fatigue $(45.4 \%)$, nausea $(42.3 \%)$, headache $(28.7 \%)$, thrombocytopenia $(28.7 \%)$, and constipation $(25.5 \%) .{ }^{18}$ The main grade 3 or 4 toxicities seen with T-DM1 were thrombocytopenia (12.9\%) with $2 \%$ of patients discontinuing the treatment, increased bleeding risk $(29.8 \%$ for T-DM1 versus $15.8 \%$ for lapatinib plus capecitabine) and increased serum transaminases with three patients discontinuing treatment due to this. ${ }^{17}$

Another Phase III trial, MARIANNE, has recently closed for recruitment and its results are awaited. It is examining T-DM1 plus placebo versus T-DM1 plus pertuzumab versus trastuzumab plus a taxane. ${ }^{19}$

\section{EGFR inhibitors}

EGFR has previously been found to promote tumor cell proliferation. EGFR is also more commonly expressed in triple negative breast cancers and is associated with a poor prognosis. ${ }^{20} \mathrm{~A}$ number of EGFR inhibitors have been extensively 
investigated as both monotherapies or in combination with other treatments.

\section{Gefitinib}

Gefitinib is a selective small molecule inhibitor of EGFR, which blocks signaling pathways involved in cell proliferation and growth.

Baselga et al performed a Phase II study examining the efficacy and pharmacodynamics of $500 \mathrm{mg}$ gefitinib daily in 31 patients with MBC which had progressed through one or two previous lines of treatment, with at least $50 \%$ of patients having tumors which were EGFR receptor positive. ${ }^{21}$ Despite finding that at that dose all the tumor biopsies analyzed (16 samples at day 28) had EGFR phosphorylation completely inhibited, none of the patients had CR or PR. SD was observed in $38.7 \%$ (12/31) patients and median time to progression (TTP) was 55 days (95\% CI 42-88). ${ }^{21}$ Other studies examining gefitinib monotherapy had a comparable low response rate. ${ }^{22-24}$

Gefitinib has also been investigated in combination with other agents. In a study by Ciardiello et al, ${ }^{25}$ patients received gefitinib $250 \mathrm{mg}$ daily with three cycles of docetaxel either at $75 \mathrm{mg} / \mathrm{m}^{2}$ or $100 \mathrm{mg} / \mathrm{m}^{2}$, increasing to six cycles if they had SD/PR/CR after three cycles. Those who had a response continued on gefitinib monotherapy until disease progression or unacceptable toxicity. They found an ORR of $54 \%$, $22 / 41$ patients (95\% CI 45\%-75\%). Interestingly, the majority of responses occurred in estrogen receptor (ER) positive $(\mathrm{ER}+)$ tumors $(70 \%$ of ER+ versus $21 \% \mathrm{ER} ; P=0.01)$. The main toxicities were grade 3 or 4 neutropenia in $49 \%$ of patients, diarrhea in $10 \%$, and rash in $5 \%$. This is in contrast to a study by Engebraaten et al which terminated early due to toxicities including diarrhea and dehydration experienced with combining weekly docetaxel and gefitinib. ${ }^{26}$

In the neoadjuvant setting Smith et al examined whether adding gefitinib to anastrozole would overcome development of endocrine resistance through observing changes to Ki67 during treatment. ${ }^{27}$ They found no clinical/biological effect. Carlson et $\mathrm{al}^{28}$ investigated adding gefitinib to fulvestrant $(n=69)$ or anastrozole $(n=73)$ as treatment for endocrine therapy naive patients with MBC. They found that the anastrozole arm had greater efficacy with an OS of 30.2 versus 23.8 months in the fulvestrant arm. ${ }^{28}$

\section{Erlotinib}

Erlotinib binds intracellularly to the adenosine triphosphate binding site of the EGFR receptor, blocking downstream signaling and therefore inhibiting cell proliferation and inducing apoptosis..$^{29,30}$ A number of Phase II studies examining erlotinib have been conducted and are summarized in Table $1 .^{29-33}$ There are currently no Phase III trials examining the role of erlotinib in breast cancer.

\section{Cetuximab}

Two Phase II trials have examined cetuximab; a monoclonal antibody against EGFR. Hobday et al enrolled 19 patients with MBC previously treated with a taxane/ anthracycline to receive cetuximab $400 \mathrm{mg} / \mathrm{m}^{2}$ in combination with irinotecan. ${ }^{34}$ The ORR was $11 \%(95 \%$ CI 1\%-33\%) with 12 patients progressing within two cycles; therefore the study terminated early. ${ }^{34}$ Carey et al randomized 102 patients with triple negative $\mathrm{MBC}$ to receive either cetuximab alone $(\mathrm{n}=31)$ or in combination with carboplatin $(n=71) .{ }^{35}$ In the cetuximab monotherapy

Table I Phase II studies examining erlotinib

\begin{tabular}{|c|c|c|c|c|c|c|c|}
\hline Study & $\begin{array}{l}\text { Treatment } \\
\text { arms }\end{array}$ & $\begin{array}{l}\text { Number per } \\
\text { arm }\end{array}$ & $\begin{array}{l}\text { ORR } \\
\text { (CR/PR) }\end{array}$ & $\begin{array}{l}\text { CBR } \\
\text { (CR/PR/SD) }\end{array}$ & $\begin{array}{l}\text { Median PFS/TTP } \\
\text { (weeks) }\end{array}$ & $\begin{array}{l}\text { Median OS } \\
\text { (months) }\end{array}$ & Comment \\
\hline $\begin{array}{l}\text { Dickler } \\
\text { et } \mathrm{al}^{30}\end{array}$ & Erlotinib/bevacizumab & 37 & $3 \%$ & $14 \%^{\mathrm{a}}$ & 11 & - & \\
\hline $\begin{array}{l}\text { Kaur } \\
\text { et } \mathrm{al}^{27}\end{array}$ & Erlotinib/weekly docetaxel & 31 & $55 \%{ }^{\mathrm{b}}$ & $90 \%{ }^{\mathrm{b}}$ & - & $23^{b}$ & $\begin{array}{l}\text { Dose reduced due } \\
\text { to hematological } \\
\text { toxicity }\end{array}$ \\
\hline $\begin{array}{l}\text { Montagna } \\
\text { et } \mathrm{al}^{28}\end{array}$ & $\begin{array}{l}\text { Metronomic capecitabine } \\
\text { and cyclophosphamide plus } \\
\text { bevacizumab and erlotinib }\end{array}$ & 26 & $62 \%^{c}$ & $75 \%{ }^{c}$ & TTP 43 & - & \\
\hline $\begin{array}{l}\text { Twelves } \\
\text { et } \mathrm{al}^{29}\end{array}$ & $\begin{array}{l}\text { Capecitabine/docetaxel/ } \\
\text { erlotinib }\end{array}$ & 24 & $67 \%^{\mathrm{d}}$ & - & - & - & $\begin{array}{l}\text { Dosing varied } \\
\text { between three arms }\end{array}$ \\
\hline $\begin{array}{l}\text { Graham } \\
\text { et } \mathrm{al}^{33}\end{array}$ & Gemcitabine/erlotinib & 59 & $14 \%$ & - & 2.8 months & - & \\
\hline
\end{tabular}

Notes: a At 26 weeks on the study; ' 20 patients evaluable; ' 24 patients evaluable; ${ }^{\mathrm{d}} 2 \mathrm{I}$ patients evaluable.

Abbreviations: CBR, clinical benefit rate; CR, complete response; ORR, objective response rate; OS, overall survival; PFS/TTP, progression free survival/time to progression; $\mathrm{PR}$, partial response; SD, stable disease for 6 months. 
arm ORR was $6 \%$ compared with $17 \%$ in the combined arm. ${ }^{35}$ Median OS was 7.5 months (95\% CI 5.0-11.6) and 10.4 months (95\% CI 7.7-13.1) respectively. Both arms were well tolerated with the main AEs being rash, infusion reactions, and fatigue. ${ }^{35}$

Based on the evidence from Baselga, ${ }^{21}$ it may be that not all tumors may be EGFR-dependent despite having EGFR receptors. To date, studies of EGFR inhibitors in breast cancer have been disappointing.

\section{HER I/HER2 dual inhibitors Lapatinib}

Lapatinib is a dual tyrosine kinase inhibitor of both HER1 and HER2. It is thought that inhibiting both pathways overcomes tumor resistance to blockade of HER2 alone by agents such as trastuzumab.

Three Phase III studies have been conducted examining lapatinib in combination with other treatments. ${ }^{36-39}$ The international, multicenter, open-label NeoALTTO trial randomized women with HER $2+$ early stage, $>2 \mathrm{~cm}$ disease in the neoadjuvant setting to receive either lapatinib $(\mathrm{n}=154)$, trastuzumab $(\mathrm{n}=149)$ or combination treatment for 6 weeks followed by 12 weeks with the addition of paclitaxel. ${ }^{37}$ Patients had definitive surgery within 4 weeks of completion of treatment. The combination arm achieved significantly better pathological complete response (pCR) $51.3 \%(95 \%$ CI $43.1-59.5 P=0.0002$ versus trastuzumab alone) than the trastuzumab arm $29.5 \%(22.4-37.5)$ or lapatinib arm 24.7\% (18.1-32.3 $P=0.74$ compared with trastuzumab arm) suggesting that dual-targeting of the HER2 receptor may be a superior approach. Because the primary endpoint of the study was $\mathrm{pCR}$ at time of surgery, whether higher $\mathrm{pCR}$ rates correlate with improved survival for this population of women is uncertain. The combination was, however, well tolerated though rates of discontinuation of treatment were higher in the lapatinib containing arms and were mainly due to excess diarrhea and transaminitis. ${ }^{37}$

The MA31 Phase III trial has recently published its interim analysis comparing taxane plus lapatinib to taxane plus trastuzumab as first line treatment of HER+ MBC. ${ }^{38}$ At median follow up of 13.6 months, data were available for 636 patients which showed a decreased PFS with the lapatinib combination versus the trastuzumab combination median PFS 8.8 months versus 11.4 months $(\mathrm{HR}=1.33$; 95\% CI $1.06-1.67 ; P=0.01) .{ }^{38}$

Geyer et al conducted a Phase III study randomizing women with locally advanced (LA) or metastatic HER2+ breast cancer which had progressed following a regimen which included an anthracycline, a taxane, and trastuzumab, to receive capecitabine alone $(n=163)$ or capecitabine and lapatinib $(\mathrm{n}=161) .{ }^{36}$ After 121 disease progression events, an interim analysis found that the combination group was superior with 49 events compared with 72 in the capecitabine monotherapy group HR 0.49 (95\% CI $0.34-0.71 ; P<0.001)$. Median TTP was 8.4 months in the combination group compared with 4.4 months for monotherapy $(P<0.001)$. ORR was 22\% (95\% CI 16\%-29\%) with combination therapy versus $14 \%(95 \%$ CI 9\%-21\%) with monotherapy $(P=0.09) .{ }^{36}$ AEs did not lead to significantly more treatment discontinuations in the combination arm, although more diarrhea, rash, and dyspepsia were seen. ${ }^{36}$ It demonstrated that targeting of the HER2 receptor beyond progression was a useful treatment option and the combination of lapatinib and capecitabine is one standard of care.

This study also demonstrated a lower risk of developing brain metastases (a major clinical problem for patients with HER2+ breast cancer) as the first site of disease progression on the combination arm (4 versus 13 months, $P=0.045) .{ }^{39} \mathrm{It}$ is thought that as it is a small molecule, lapatinib may cross the blood-brain barrier more effectively than other larger agents. A Phase II study investigated the use of lapatinib in patients with HER2+ breast cancer who had progressive brain metastases and had been previously treated with trastuzumab and whole brain radiotherapy. ${ }^{40}$ The ORR was only $6 \%$ but a fifth of patients had a volumetric reduction of $>20 \%$ in the volume of their brain metastases. On progression, patients were treated with lapatinib and capecitabine in combination with an ORR of $20 \%$, though this improvement may have been due to capecitabine alone.

The CEREBEL trial examined the use of lapatinib as prophylaxis against brain metastases in patients with HER2+ $\mathrm{MBC}$ and no central nervous system (CNS) involvement at baseline. ${ }^{41}$ Patients were randomized to receive either trastuzumab plus capecitabine $(\mathrm{n}=218)$ or lapatinib plus capecitabine $(n=218)$. Approximately $40 \%$ of patients had not received prior trastuzumab. The primary endpoint of CNS relapse was 3\% for the lapatinib arm and $4 \%$ for trastuzumab. However, both median PFS and OS were reduced in the lapatinib arm compared with trastuzumab; therefore the study was terminated early. ${ }^{41}$ The efficacy of lapatinib in patients with brain metastases remains of interest, but uncertain. An ongoing study in the United Kingdom (LANTERN) is randomizing patients post radiotherapy for brain metastases to lapatinib and capecitabine or trastuzumab and capecitabine, which may provide further evidence. 


\section{Neratinib}

Neratinib is an irreversible pan ErbB tyrosine kinase receptor inhibitor. A Phase II study by Burstein et $\mathrm{a}^{42}$ compared patients with $\mathrm{MBC}$ who had received prior trastuzumab therapy $(\mathrm{n}=66)$ and those who were trastuzumab naive $(\mathrm{n}=70)$. They were given $240 \mathrm{mg}$ neratinib and the primary endpoint of PFS at 16 weeks was evaluated. ORR was $24 \%$ in pre-treated patients (95\% CI 14\%-36\%) and 56\% in trastuzumab naive patients (95\% CI 43\%-69\%). The treatment was generally well tolerated with diarrhea as the main grade 3/4 AE. Diarrhea improved as patients received further weeks of treatment. Further studies are ongoing.

\section{mTOR inhibitors}

The mTOR protein kinase integrates signaling from Ras and phosphatidylinositol-3-OH kinase (PI3K) resulting in further phosphorylation of downstream proteins in growth signaling pathways which, when behaving abhorrently, drive tumorigenesis. ${ }^{43}$ Furthermore, mTOR signaling pathways are thought to contribute to anticancer drug resistance. ${ }^{44}$

\section{Everolimus}

Everolimus inhibits mTOR through allosteric binding to mTOR complex 1 (mTORC1). Phase II studies have investigated it in combination with hormone therapy in order to exploit its potential for overcoming hormone resistance. . $^{45,46}$ The BOLERO-2 Phase III randomized study compared exemestane in combination with placebo $(\mathrm{n}=239)$ or everolimus $(n=485)$ in women with hormone receptor positive in advanced breast cancer which had progressed while receiving prior nonsteroidal aromatase inhibitors. ${ }^{47}$ The investigators found that the combination treatment had improved median PFS (based on central assessment) of 10.6 months versus 4.1 months with placebo (HR $0.36 ; 95 \%$ CI $0.27-0.47$; $P<0.001) .{ }^{47}$ Data for OS are yet to be presented, although at 12.5 months there were fewer deaths reported with combination treatment; $17.2 \%$ versus $22.7 \%$ with placebo. ${ }^{48}$ The improved PFS was at the expense of more grade III toxicities for the combination arm with $19 \%$ of patients on the combination arm discontinuing treatment compared to $3 \%$ on the placebo/exemestane. The commonest grade III toxicity was stomatitis ( $8 \%$ versus $1 \%)$.

As previously stated, mTOR inhibitors may also be effective at overcoming drug resistance to other anti-cancer therapies. BOLERO-1 is looking at the use of everolimus in addition to trastuzumab and paclitaxel in patients with metasatic HER2+ cancer in the first line setting, with BOLERO-3 randomizing patients with metastatic HER2+ breast cancer who have received no more than three lines of cytotoxic therapy to trastuzumab and vinorelbine with or without everolimus. Results from these studies are awaited.

\section{Temsirolimus}

Although Phase II studies showed some antitumor activity and a tolerable safety profile, HORIZON, the recent Phase III study of temsirolimus versus placebo in combination with letrozole, was terminated early by the independent data monitoring committee due to lack of efficacy. Patients with hormone receptor positive $\mathrm{MBC}$ and no prior exposure to aromatase inhibitors were randomized to receive letrozole in combination with either temsirolimus or placebo. There was no improvement in median PFS with the addition of temsirolimus (HR 0.90 95\% CI 0.76-1.07), however more grade $3 / 4$ events were seen ( $37 \%$ versus $24 \%$ for placebo). ${ }^{49}$ Trials of temsirolimus in combination with other drugs are ongoing.

\section{PI3K inhibitors}

The PI3K pathway plays an essential role in cell survival, differentiation and growth. ${ }^{50} \mathrm{PI} 3 \mathrm{Ks}$ are activated by cell membrane receptors such as HER2 and insulin-like growth factor receptor resulting in phosphorylation of phosphatidylinositol bisphosphate to phosphatidylinositol triphosphate. ${ }^{50}$ This enables Protein kinase B or AKT to bind, which activates mTOR resulting in further downstream signaling. Mutations in PI3K are found in $20 \%-30 \%$ of breast cancers, most frequently in hormone receptor positive cancers and less commonly in triple negative tumors. ${ }^{51}$

In addition, there may be an association between PI3K mutations and hormone therapy resistance, ${ }^{52}$ which is being investigated further in the Phase I/II study randomizing patients to receive letrozole in combination with XL147 or XL765. ${ }^{50}$ Phase I/II trials of other PI3K inhibitors are also currently recruiting.

\section{Ras inhibitors \\ Tipifarnib}

Growth factor receptor pathways are integral to cellular signal transduction and growth. A component of these pathways is the attachment of Ras proteins to cell membranes, which enables interaction with membrane receptors and downstream signaling to occur. In order to attach to cellular membranes, new Ras proteins must be modified by farnesylation which involves covalent attachment of farnesyl to a COOH-terminal amino acid sequence on the Ras protein. ${ }^{53}$ This process is catalyzed by farnesyl protein transferase which is inhibited by tipifarnib. In addition, tipifarnib inhibits estrogen signaling pathways and theoretically may overcome resistance to hormone therapies. ${ }^{54}$ Therefore, many of the Phase II trials in the metastatic 
setting have combined tipifarnib with hormone therapy (see Table 2). ${ }^{54-57}$ Phase III trials are yet to be conducted.

\section{PARP inhibitors}

Poly(ADP-ribose) polymerase (PARP) is an enzyme responsible for repair of single stranded DNA breaks. Olaparib is a PARP inhibitor which has been investigated in two Phase II studies. Gelmon et al treated 26 patients with advanced triple-negative breast cancer with olaparib $400 \mathrm{mg}$ twice daily. ${ }^{58}$ There were no ORs, however, in patients who had BRCA mutated cancers; $63 \%(5 / 8)$ had SD for $>8$ weeks. For those who were BRCA wild type, 30\% (7/23) had SD. The most common AEs reported were fatigue, decreased appetite, nausea, and vomiting. ${ }^{58}$

In another Phase II study examining olaparib in 56 women with BRCA1 or BRCA2 positive breast cancers, an ORR of $41 \%$ (95\% CI 25\%-59\%) was achieved in patients receiving a $400 \mathrm{mg}$ twice daily dose (11/27) and 22\% (95\% CI $11 \%-41 \%)$ in those receiving $100 \mathrm{mg}$ twice daily $(7 / 27) .59$ Similar AEs were reported and were mainly low grade. A Phase III trial has yet to be developed in breast cancer. Another inhibitor, iniparib which was initially thought to act on PARP, did not show significant improvement of PFS or OS when combined with carboplatin/gemcitabine in a Phase III study in women with pretreated MBC. ${ }^{60}$ It has subsequently been demonstrated that iniparib is not an inhibitor of PARP and its development has been put on hold.

\section{VEGF inhibitors}

Tumor angiogenesis is thought to play a critical role in the growth and metastasis of tumors in order to supply cancerous cells with the nutrients and oxygen they need to survive. VEGF mediates formation of blood vessels and is known to be over expressed in breast cancer cells (see Figure 2). ${ }^{61}$

\section{Bevacizumab}

Bevacizumab is a monoclonal antibody which blocks VEGF from binding to its receptor on endothelial cells inhibiting their proliferation. ${ }^{62}$ To date, unfortunately, clinical trials using the drug in breast cancer have been relatively disappointing. In the Phase III setting, four large randomized trials have been conducted. ${ }^{63-66}$

The AVADO trial randomized 736 patients with a local recurrence or metastatic HER2 negative breast cancer or on an intention to treat (ITT) basis to receive docetaxel with either placebo or bevacizumab at 7.5 or $15 \mathrm{mg} / \mathrm{kg}$ doses. ${ }^{63}$ Bevacizumab at $15 \mathrm{mg} / \mathrm{kg}$ combined with docetaxel showed a statistically significant (but clinically small) improvement in PFS of median 10.1 months versus 8.2 months with docetaxel plus placebo (HR $0.7795 \% \mathrm{CI}$ 0.64-0.93 $P=0.006) .{ }^{63}$ AEs of hypertension, bleeding, and proteinuria were more commonly seen in the bevacizumab arm, however there were similar numbers of AEs leading to death in both arms ( $2 \%$ in both bevacizumab arms, $3 \%$ placebo arm). ${ }^{63}$

Another open-label trial randomized 722 women with MBC to receive either paclitaxel $(n=354)$ or paclitaxel plus bevacizumab $(n=368)$ as first line treatment (prior adjuvant chemotherapy/hormone therapy/trastuzumab was allowed). ${ }^{66}$ The primary endpoint was PFS and after 624 events, the combined arm had a median PFS of 11.8 months versus 5.6 months in the paclitaxel monotherapy arm (HR $0.60, P<0.001$ ). ORR was $36.9 \%$ for the combination compared to $21.2 \%$, for paclitaxel $(P<0.001){ }^{66}$ Importantly, neither study showed an improvement in OS with the addition of bevacizumab to standard chemotherapy.

Miller et al conducted an open label study which randomized 462 patients with heavily pretreated MBC equally to receive capecitabine alone or in combination with bevacizumab. ${ }^{64}$ Although they found that there were increased ORRs with the addition of bevacizumab (19.8\% versus $9.1 \%, P=0.001)$, this did not translate into improved PFS (4.9 versus 4.2 months, HR 0.98 ) or OS (15.1 versus 14.5 months). The only more frequent grade 3 or 4 toxicity with the combined arm was hypertension. ${ }^{64}$

Table 2 Phase II studies of tipifarnib in MBC

\begin{tabular}{|c|c|c|c|c|c|}
\hline Study & Treatment arms & $\begin{array}{l}\text { Number of patients } \\
\text { per arm }\end{array}$ & $\begin{array}{l}\text { ORR } \\
\text { (CR/PR) }\end{array}$ & CBR & $\begin{array}{l}\text { Median PFS/TTP } \\
\text { (months) }\end{array}$ \\
\hline Dalenc et $\mathrm{al}^{54}$ & Tipifarnib/tamoxifen & 20 & $5 \%$ & $50 \%$ & 5.7 \\
\hline Li et $\mathrm{al}^{55}$ & Tipifarnib/capecitabine & 63 & $9.5 \%$ & $32 \%$ & 2.6 \\
\hline Li et $\mathrm{al}^{56}$ & Tipifarnib/fulvestrant & 31 & $35.5 \%$ & $51.6 \%$ & TTP 7.2 \\
\hline \multirow[t]{2}{*}{ Johnston et al ${ }^{57}$} & Tipifarnib/letrozole & 80 & $30 \%$ & $49 \%$ & TTP 5.6 \\
\hline & Letrozole/placebo & 40 & $38 \%$ & $62 \%$ & TTP I0.8 \\
\hline
\end{tabular}

Abbreviations: $C B R$, clinical benefit rate; CR, complete response; MBC, metastatic breast cancer; ORR, objective response rate; PFS/TTP, progression free survival/time to progression; PR, partial response. 


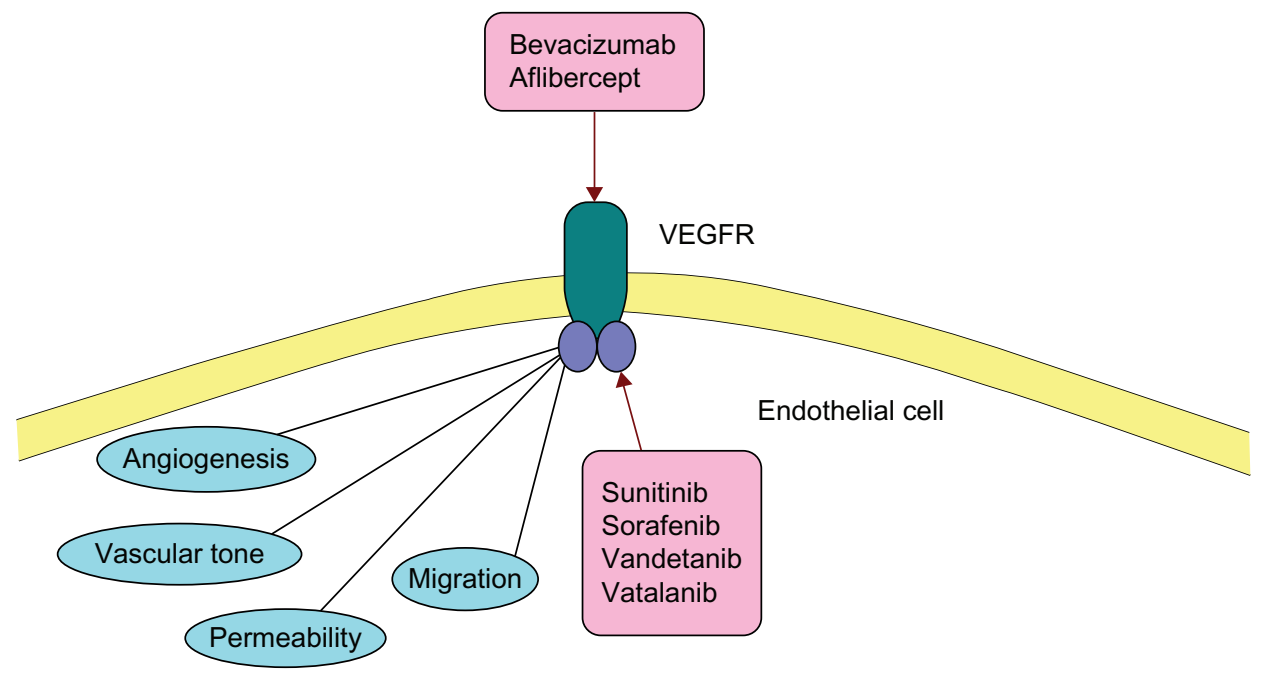

Figure 2 Role of VEGFR (vascular endothelial growth factor receptor) in vascularization/angiogenesis and its inhibitors.

The RIBBON-1 study examined whether the addition of bevacizumab to chemotherapy as first line treatment in locally advanced or metastatic HER 2 negative breast cancer improved efficacy. ${ }^{65}$ Before 2:1 randomization, investigators allocated a patient to receive either capecitabine $(n=615)$, a taxane based regimen $(\mathrm{n}=307)$, or an anthracycline based combination $(n=315)$. The addition of bevacizumab improved PFS; in the capecitabine group PFS was 6.2 months with placebo versus 9.8 months with bevacizumab (HR, 0.68 ; 95\% CI 0.54-0.86), in the taxane/anthracycline group PFS was 8.3 months with placebo versus 10.7 months with bevacizumab (HR 0.77 ; $95 \%$ CI $0.60-0.99) .{ }^{65}$ There was no significant difference in 1 year OS. ${ }^{65}$ RIBBON-2 examined the addition of bevacizumab to chemotherapy as second line treatment in the setting of HER2 negative locally advanced or MBC; 684 patients were randomized to receive chemotherapy and placebo $(n=225)$ or chemotherapy and bevacizumab $(\mathrm{n}=459) .{ }^{67}$ The median PFS was improved from 5.1 months with placebo to 7.2 months with bevacizumab (HR $0.78 P=0.0072){ }^{67}$

There are a number of trials not yet reported, examining bevacizumab in the adjuvant setting based on the hypothesis that anti-angiogenic agents may be most effective as adjuvant treatment through preventing angiogenesis before metastases are established. E5103 is examining how the use of bevacizumab plus taxane and anthracycline chemotherapy after surgery may reduce the risk of recurrence and whether further bevacizumab maintenance adds benefit. BETH has randomized patients with HER2+ cancer to receive trastuzumab and standard chemotherapy plus bevacizumab or placebo. Preliminary results of the
BEATRICE trial looking at the addition of bevacizumab to anthracycline/taxane based chemotherapy as adjuvant treatment for triple negative early breast cancer were recently presented at the San Antonio Breast cancer Symposium. At a median follow-up of 32 months, the hazard ratio for invasive disease-free survival was 0.87 (95\% CI $0.72-1.07$ ) in favor of patients assigned to chemotherapy and bevacizumab randomizes patients with triple negative breast cancer to receive standard chemotherapy plus placebo or bevacizumab as adjuvant therapy following surgery. ${ }^{68}$

\section{Aflibercept}

Aflibercept is a soluble decoy protein which has high affinity for the family of VEGF which bind to VEGFR-1 and VEGFR-2. ${ }^{20}$ The only Phase II study reported was terminated early due to lack of efficacy and a PFS of only 2.4 months. $^{20}$

\section{Multikinase inhibitors Sorafenib}

Sorafenib is a multikinase inhibitor which targets a number of receptors including VEGF receptor-1 (VEGFR-1), VEGFR-2, VEGFR-3, Flt-3, platelet-derived growth factor receptor (PDGFR), Raf kinase, and c-Kit producing both anti-angiogenesis and anti-proliferative effects. Two Phase II studies have examined its potential efficacy in breast cancer. Moreno-Aspitia et al examined the effect on tumor response of sorafenib monotherapy. ${ }^{69}$ None of the 20 patients assessable for response achieved a CR/PR although two had SD for $>6$ months. ${ }^{69}$ 
Baselga et al randomized 229 patients with HER2 negative $\mathrm{LA} / \mathrm{MBC}$ to receive capecitabine plus placebo or sorafenib. They found that sorafenib significantly improved PFS; 6.4 months versus 4.1 months (HR 0.58 ; 95\% CI 0.41 to $0.81 ; P=0.001){ }^{70}$ Toxicities of sorafenib at a dose of $400 \mathrm{mg}$ twice daily were unacceptable with $20 \%$ of patients discontinuing due to AEs; therefore it was recommended that a lower dose be used in future studies. ${ }^{70}$

\section{Sunitinib}

Sunitinib is also a multikinase inhibitor, blocking VEGFR, ckit, and PDGFR. Three Phase III trials have investigated its use in breast cancer. Barrios et al conducted an open label trial comparing sunitinib with capecitabine in HER2 negative advanced disease. The trial was terminated early when the independent review committee deemed that it would not reach its primary endpoint of improved PFS with sunitinib compared to capecitabine. ${ }^{71}$ Nor was PFS or OS improved when used in combination with capecitabine versus capecitabine alone in another Phase III trial randomizing patients with HER2+/- advanced disease. ${ }^{72}$ Finally, Bergh et al also reported negative results in their Phase III study in patients with HER2- LA/MBC comparing sunitinib in combination with paclitaxel $(n=296)$ versus paclitaxel alone $(\mathrm{n}=297) \cdot{ }^{73}$ Although ORR was significantly increased with the addition of sunitinib $(55 \%$ versus $42 \% P=0.001$ ), there was no significant increase in PFS or OS. ${ }^{73}$ RESILIENCE, a Phase III trial examining sunitinib in combination with capecitabine is currently recruiting.

\section{Vandetanib}

Vandetanib is an inhibitor of VEGFR and EGFR as well as RET tyrosine kinase. Two trials have investigated it in the Phase II setting and further studies are ongoing. Miller et al enrolled 46 patients with previously treated MBC to receive vandetanib in a dose-finding study. ${ }^{74}$ They found that it was well tolerated with the main toxicities reported as rash, prolongation of QTc interval, and diarrhea which appeared to be dose dependent. There were no ORs and one patient had SD for $>24$ weeks.

Boér et al randomized 64 patients with pretreated MBC on an ITT basis to receive docetaxel with vandetanib $(n=35)$ or docetaxel with placebo $(n=29) .{ }^{75}$ They found no clinical benefit for the addition of vandetanib, however it was generally reasonably tolerated with 15 patients discontinuing due to AEs including diarrhea and neutropenia in the vandetanib arm compared to 10 with placebo. ${ }^{75}$

\section{Vatalanib}

Vatalanib is a VEGFR inhibitor and at higher concentrations inhibits other tyrosine kinases such as PDGF, c-kit, and c-Fms. ${ }^{76}$ The Hoosier oncology group conducted a Phase I/II study of vatalanib in combination with trastuzumab in patients with HER2+ MBC, but the trial was terminated due to low patient enrolment and toxicities. ${ }^{77}$

\section{Future aims}

The evolution of targeted therapies is an important step in the creation of individualized cancer management. The possible targets for therapies multiply, at the same time as our understanding of the mechanisms underlying cancer improves. However, better understanding of which patients will gain most benefit from targeted treatments is still required. Focus is currently on developing robust biomarkers in order to aid prediction of response so that in future, patients will receive only treatments that will confer an advantage.

\section{Disclosure}

Dr Wardley has been paid honoraria from Celgene, Easai, GlaxoSmithKline, Novartis and Roche and has received travel assistance from Roche. The authors have no other conflicts of interest in this work.

\section{References}

1. Schechter AL, Stern DF, Vaidyanathan L, et al. The neu oncogene: an erb-B-related gene encoding a 185,000-Mr tumour antigen. Nature. 1984;312(5994):513-516.

2. Slamon D, Godolphin W, Jones L, et al. Studies of the HER-2/neu proto-oncogene in human breast and ovarian cancer. Science. 1989; 244(4905):707-712.

3. Ross JS, Slodkowska EA, Symmans WF, Pusztai L, Ravdin PM, Hortobagyi GN. The HER-2 receptor and breast cancer: ten years of targeted anti-HER-2 therapy and personalized medicine. The Oncologist. 2009;14(4):320-368.

4. Hudis CA. Trastuzumab - mechanism of action and use in clinical practice. N Engl J Med. 2007;357(1):39-51.

5. Perez EA, Romond EH, Suman VJ, et al. Updated results of the combined analysis of NCCTG N9831 and NSABP B-31 adjuvant chemotherapy with/without trastuzumab in patients with HER2-positive breast cancer. Paper presented at: ASCO Annual Meeting Proceedings Part I 2007 San Francisco.

6. Smith I, Procter M, Gelber RD, et al. 2-year follow-up of trastuzumab after adjuvant chemotherapy in HER2-positive breast cancer: a randomised controlled trial. Lancet. 2007;369(9555): 29-36.

7. Slamon D, Eiermann W, Robert N, et al. Adjuvant trastuzumab in HER2positive breast cancer. $N$ Engl J Med. 2011;365(14):1273-1283.

8. Agus DB, Gordon MS, Taylor C, et al. Phase I clinical study of pertuzumab, a novel HER dimerization inhibitor, in patients with advanced cancer. J Clin Oncol. 2005;23(11):2534-2543.

9. Chen X, Yeung TK, Wang Z. Enhanced drug resistance in cells coexpressing ErbB2 with EGF receptor or ErbB3. Biochem Biophys Res Commun. 2000;277(3):757-763. 
10. Gianni L, Lladó A, Bianchi G, et al. Open-label, Phase II, multicenter, randomized study of the efficacy and safety of two dose levels of pertuzumab, a human epidermal growth factor receptor 2 dimerization inhibitor, in patients with human epidermal growth factor receptor 2-negative metastatic breast cancer. J Clin Oncol. 2010;28(7):1131-1137.

11. Baselga J, Gelmon KA, Verma S, et al. Phase II trial of pertuzumab and trastuzumab in patients with human epidermal growth factor receptor 2-positive metastatic breast cancer that progressed during prior trastuzumab therapy. J Clin Oncol. 2010;28(7):1138-1144.

12. Baselga J, Cortés J, Kim S-B, et al. Pertuzumab plus trastuzumab plus docetaxel for metastatic breast cancer. N Engl J Med. 2012; 366(2):109-119.

13. Swain SM, Kim SB, Cortes J, et al. Confirmatory overall survival (OS) analysis of CLEOPATRA: a randomized, double-blind, placebocontrolled Phase III study with pertuzumab (P), trastuzumab (T), and docetaxel (D) in patients (pts) with HER2-positive first-line (1L) metastatic breast cancer (MBC). Cancer Research. 2012;72(24 Suppl 3): abstract P5-18-26.

14. Lewis Phillips GD, Li G, Dugger DL, et al. Targeting HER2-positive breast cancer with trastuzumab-DM1, an antibody-cytotoxic drug conjugate. Cancer Research. 2008;68(22):9280-9290.

15. Krop IE, Beeram M, Modi S, et al. Phase I study of trastuzumab-DM1, an HER2 antibody-drug conjugate, given every 3 weeks to patients with HER2-positive metastatic breast cancer. J Clin Oncol. 2010; 28(16):2698-2704.

16. Krop IE, LoRusso P, Miller KD, et al. A Phase II study of trastuzumab emtansine in patients with human epidermal growth factor receptor 2-positive metastatic breast cancer who were previously treated with trastuzumab, lapatinib, an anthracycline, a taxane, and capecitabine. J Clin Oncol. 2012;30(26):3234-3241.

17. Verma S, Miles D, Gianni L, et al. Trastuzumab emtansine for HER2-positive advanced breast cancer. $N$ Engl J Med. 2012;367(19): 1783-1791.

18. Diéras V, Harbeck N, Budd GT, et al. Trastuzumab emtansine in HER2-positive metastatic breast cancer: pooled safety analysis from seven studies. Cancer Research. 2012;72(24 Suppl 3):P5-18-06.

19. Ellis P, Barrios C, Im Y, Patre M, Branle F, Perez E. MARIANNE: A phase III, randomized study of trastuzumab-DM1 (T-DM1) with or without pertuzumab $(\mathrm{P})$ compared with trastuzumab $(\mathrm{H})$ plus taxane for first-line treatment of HER2-positive, progressive, or recurrent locally advanced or metastatic breast cancer (MBC). J Clin Oncol. 2011;29(Suppl; abstr TPS102).

20. Alvarez RH, Valero V, Hortobagyi GN. Emerging targeted therapies for breast cancer. J Clin Oncol. 2010;28(20):3366-3379.

21. Baselga J, Albanell J, Ruiz A, et al. Phase II and tumor pharmacodynamic study of gefitinib in patients with advanced breast cancer. J Clin Oncol. 2005;23(23):5323-5333.

22. Robertson J, Gutteridge E, Cheung K, Owers R. Gefitinib (ZD1839) is active in acquired tamoxifen (TAM)-resistant oestrogen receptor (ER)positive and ER-negative breast cancer: results from a phase II study. Proc Am Soc Clin Oncol. 2003;22(abstr 23).

23. Albain K, Elledge R, Gradishar WJ, Natale RB. Open-label, phase II, multicenter trial of ZD 1839 ('Iressa') in patients with advanced breast cancer. Breast Cancer Research And Treatment. 2002;76(S33): (Suppl 1; abstr 20).

24. von Minckwitz G, Jonat W, Fasching P, et al. A multicentre phase II study on gefitinib in taxane- and anthracycline-pretreated metastatic breast cancer. Breast Cancer Res Treat. 2005;89(2):165-172.

25. Ciardiello F, Troiani T, Caputo F, et al. Phase II trial of gefitinib combined with docetaxel as first-line therapy in patients with metastatic breast cancer. Br J Cancer. 2006;94(11):1604-1609.

26. Engebraaten O, Edvardsen H, Løkkevik E, et al. Gefitinib in combination with weekly docetaxel in patients with metastatic breast cancer caused unexpected toxicity: results from a randomized Phase II clinical trial. ISRN Oncology. 2012;2012:8.

27. Smith IE, Walsh G, Skene A, et al. A Phase II placebo-controlled trial of neoadjuvant anastrozole alone or with gefitinib in early breast cancer. J Clin Oncol. 2007;25(25):3816-3822.
28. Carlson R W, O’Neill A, Vidaurre T, Gomez HL, Badve S. Randomized phase II trial of gefitinib plus anastrozole or fulvestrant in postmenopausal, metastatic breast cancer. J Clin Oncol. 2009; 27(15S): 1013

29. Twelves C, Trigo JM, Jones R, et al. Erlotinib in combination with capecitabine and docetaxel in patients with metastatic breast cancer: a dose-escalation study. Eur J Cancer. 2008;44(3):419-426.

30. Dickler MN, Rugo HS, Eberle CA, et al. A phase ii trial of erlotinib in combination with bevacizumab in patients with metastatic breast cancer. Clin Cancer Res. 2008;14(23):7878-7883.

31. Kaur H, Silverman P, Singh D, Cooper BW, Fu P, Krishnamurthi S. Toxicity and outcome data in a phase II study of weekly docetaxel in combination with erlotinib in recurrent and/or metastatic breast cancer (MBC). J Clin Oncol. 2006;24(18S):10623.

32. Montagna E, Cancello G, Bagnardi V, et al. Metronomic chemotherapy combined with bevacizumab and erlotinib in patients with metastatic HER2-negative breast cancer: clinical and biological activity. Clin Breast Cancer. 2012;12(3):207-214.

33. Graham DL, Hillman DW, Hobday TJ, et al. N0234: Phase II study of erlotinib (OSI-774) plus gemcitabine as first-or second-line therapy for metastatic breast cancer (MBC). J Clin Oncol. 2005;23(16S):644.

34. Hobday TJ, Stella PJ, Fitch TR, et al. N0436: a phase II trial of irinotecan plus cetuximab in patients with metastatic breast cancer and prior anthracycline and/or taxane-containing therapy. J Clin Oncol. 2008;26(Suppl):abstr 1081.

35. Carey LA, Rugo HS, Marcom PK, et al. TBCRC 001: randomized phase ii study of cetuximab in combination with carboplatin in Stage IV triple-negative breast cancer. J Clin Oncol. 2012;30(21):2615-2623.

36. Geyer CE, Forster J, Lindquist D, et al. Lapatinib plus Capecitabine for HER2-Positive Advanced Breast Cancer. New England Journal of Medicine. 2006;355(26):2733-2743.

37. Baselga J, Bradbury I, Eidtmann H, et al. Lapatinib with trastuzumab for HER2-positive early breast cancer (NeoALTTO): a randomised, openlabel, multicentre, phase 3 trial. The Lancet. 379(9816):633-640.

38. Gelmon KA, Boyle F, Kaufman B, et al. Open-label phase III randomized controlled trial comparing taxane-based chemotherapy (Tax) with lapatinib (L) or trastuzumab (T) as first-line therapy for women with HER2+ metastatic breast cancer: Interim analysis (IA) of NCIC CTG MA.31/GSK EGF 108919. J Clin Oncol. 2012;30(Suppl 15):abstract LBA671.

39. Cameron D, Casey M, Press M, et al. A phase III randomized comparison of lapatinib plus capecitabine versus capecitabine alone in women with advanced breast cancer that has progressed on trastuzumab: updated efficacy and biomarker analyses. Breast Cancer Res Treat. Dec 2008;112(3):533-543.

40. Lin NU, Diéras V, Paul D, et al. Multicenter Phase II Study of Lapatinib in Patients with Brain Metastases from HER2-Positive Breast Cancer. Clinical Cancer Research. February 15, 2009;15(4):1452-1459.

41. Pivot X, Semiglazov V, Żurawski B, et al. CEREBEL (EGF111438): An open label randomized phase III study comparing the incidence of CNS metastases in patients (pts) with HER2+ Metastatic Breast Cancer (MBC), treated with Lapatinib plus Capecitabine (LC) versus Trastuzumab plus Capecitabine (TC). Annals of Oncology. 2012;23(Suppl 9): Abstract LBA11.

42. Burstein HJ, Sun Y, Dirix LY, et al. Neratinib, an irreversible ErbB receptor tyrosine kinase inhibitor, in patients with advanced ErbB2positive breast cancer. J Clin Oncol. 2010;28(8):1301-1307.

43. Shaw RJ, Cantley LC. Ras, $\mathrm{PI}(3) \mathrm{K}$ and $\mathrm{mTOR}$ signalling controls tumour cell growth. Nature. 2006;441(7092):424-430.

44. Jiang BH, Liu LZ. Role of mTOR in anticancer drug resistance: perspectives for improved drug treatment. Drug Resistance Updates: Reviews and Commentaries in Antimicrobial and Anticancer Chemotherapy. 2008;11(3):63-76.

45. Bachelot T, Bourgier C, Cropet C, et al. Randomized phase II trial of everolimus in combination with tamoxifen in patients with hormone receptor-positive, human epidermal growth factor receptor 2-negative metastatic breast cancer with prior exposure to aromatase inhibitors: a GINECO Study. J Clin Oncol. 2012;30(22):2718-2724. 
46. Baselga J, Semiglazov V, van Dam P, et al. Phase II randomized study of neoadjuvant everolimus plus letrozole compared with placebo plus letrozole in patients with estrogen receptor-positive breast cancer. J Clin Oncol. 2009;27(16):2630-2637.

47. Baselga J, Campone M, Piccart M, et al. Everolimus in postmenopausal hormone-receptor-positive advanced breast cancer. $N$ Engl J Med. 2012;366(6):520-529.

48. Piccart-Gebhart MJ, Noguchi S, Pritchard KI, et al. Everolimus for postmenopausal women with advanced breast cancer: Updated results of the BOLERO-2 phase III trial. J Clin Oncol. 2012;30(Suppl):abstr 559.

49. Wolff AC, Lazar AA, Bondarenko I, et al. Randomized phase III placebo-controlled trial of letrozole plus oral temsirolimus as first-line endocrine therapy in postmenopausal women with locally advanced or metastatic breast cancer. J Clin Oncol. 2013;31(2):195-202.

50. Baselga J. Targeting the phosphoinositide-3 (PI3) kinase pathway in breast cancer. Oncologist. 2011;16(Suppl 1):12-19.

51. Stemke-Hale K, Gonzalez-Angulo AM, Lluch A, et al. An integrative genomic and proteomic analysis of PIK3CA, PTEN, and AKT mutations in breast cancer. Cancer Research. 2008;68(15):6084-6091.

52. Tokunaga E, Kimura Y, Mashino K, et al. Activation of PI3K/Akt signaling and hormone resistance in breast cancer. Breast Cancer. 2006;13(2):137-144

53. End DW, Smets G, Todd AV, et al. Characterization of the antitumor effects of the selective farnesyl protein transferase inhibitor R115777 in vivo and in vitro. Cancer Research. 2001;61(1):131-137.

54. Dalenc F, Doisneau-Sixou SF, Allal BC, et al. Tipifarnib plus tamoxifen in tamoxifen-resistant metastatic breast cancer: a negative phase II and screening of potential therapeutic markers by proteomic analysis. Clin Cancer Res. 2010;16(4):1264-1271.

55. Li T, Guo M, Gradishar W, et al. A phase II trial of capecitabine in combination with the farnesyltransferase inhibitor tipifarnib in patients with anthracycline-treated and taxane-resistant metastatic breast cancer: an Eastern Cooperative Oncology Group Study (E1103). Breast Cancer Res Treat. 2012;134(1):345-352.

56. Li T, Christos PJ, Sparano JA, et al. Phase II trial of the farnesyltransferase inhibitor tipifarnib plus fulvestrant in hormone receptor-positive metastatic breast cancer: New York Cancer Consortium Trial P6205. Ann Oncol. 2009;20(4):642-647.

57. Johnston S, Semiglazov V, Manikhas G, et al. A phase II, randomized, blinded study of the farnesyltransferase inhibitor tipifarnib combined with letrozole in the treatment of advanced breast cancer after antiestrogen therapy. Breast Cancer Res Treat. 2008;110(2):327-335.

58. Gelmon KA, Tischkowitz M, Mackay H, et al. Olaparib in patients with recurrent high-grade serous or poorly differentiated ovarian carcinoma or triple-negative breast cancer: a phase 2, multicentre, openlabel, non-randomised study. Lancet Oncol. 2011;12(9):852-861.

59. Tutt A, Robson M, Garber JE, et al. Oral poly(ADP-ribose) polymerase inhibitor olaparib in patients with BRCA1 or BRCA2 mutations and advanced breast cancer: a proof-of-concept trial. Lancet. 376(9737):235-244.

60. O'Shaughnessy J, Schwartzberg LS, Danso MA, et al. A randomized phase III study of iniparib (BSI-201) in combination with gemcitabine/ carboplatin $(\mathrm{G} / \mathrm{C})$ in metastatic triple-negative breast cancer (TNBC). J Clin Oncol. 2011;29(Suppl 15):abstr 1007.

61. Relf M, LeJeune S, Scott PAE, et al. Expression of the angiogenic factors vascular endothelial cell growth factor, acidic and basic fibroblast growth factor, tumor growth factor $\beta-1$, platelet-derived endothelial cell growth factor, placenta growth factor, and pleiotrophin in human primary breast cancer and its relation to angiogenesis. Cancer Res. 1997;57(5):963-969.

62. Shih T, Lindley C. Bevacizumab: an angiogenesis inhibitor for the treatment of solid malignancies. Clin Ther. 2006;28(11):1779-1802.

63. Miles DW, Chan A, Dirix LY, et al. Phase III Study of Bevacizumab Plus Docetaxel Compared With Placebo Plus Docetaxel for the First-Line Treatment of Human Epidermal Growth Factor Receptor 2-Negative Metastatic Breast Cancer. J Clin Oncol. 2010;28(20):3239-3247.
64. Miller KD, Chap LI, Holmes FA, et al. Randomized Phase III Trial of Capecitabine Compared With Bevacizumab Plus Capecitabine in Patients With Previously Treated Metastatic Breast Cancer. J Clin Oncol. 2005;23(4):792-799.

65. Robert NJ, Diéras V, Glaspy J, et al. RIBBON-1: randomized, doubleblind, placebo-controlled, phase iii trial of chemotherapy with or without bevacizumab for first-line treatment of human epidermal growth factor receptor 2-negative, locally recurrent or metastatic breast cancer. J Clin Oncol. 2011;29(10):1252-1260.

66. Miller K, Wang M, Gralow J, et al. Paclitaxel plus bevacizumab versus paclitaxel alone for metastatic breast cancer. $N$ Engl J Med. 2007;357(26):2666-2676.

67. Brufsky A, Hurvitz SA, Perez EA, et al. Final overall survival (OS) and safety analyses of RIBBON-2, a randomised phase III trial of bevacizumab $(\mathrm{BEV})$ versus placebo $(\mathrm{PL})$ combined with second line chemotherapy (CT) for HER2-negative-BEV-naive metastatic breast cancer (MBC). J Clin Oncol. 2012;30(Suppl 27):abstr 100.

68. Cameron D, Brown J, Dent R, et al. Primary results of BEATRICE, a randomized phase III trial evaluating adjuvant bevacizumabcontaining therapy in triple-negative breast cancer. Cancer Research. 2012;72(24, Suppl 3). Available from: http://cancerres.aacrjournals. org/cgi/content/short/72/24_MeetingAbstracts/S6-5?rss=1. Accessed May 20, 2013.

69. Moreno-Aspitia A, Morton RF, Hillman DW, et al. Phase II trial of sorafenib in patients with metastatic breast cancer previously exposed to anthracyclines or taxanes: North Central Cancer Treatment Group and Mayo Clinic Trial N0336. J Clin Oncol. 2009;27(1):11-15.

70. Baselga J, Segalla JGM, Roché H, et al. Sorafenib in combination with capecitabine: an oral regimen for patients with HER2-negative locally advanced or metastatic breast cancer. J Clin Oncol. 2012; 30(13):1484-1491.

71. Barrios CH, Liu MC, Lee SC, et al. Phase III randomized trial of sunitinib versus capecitabine in patients with previously treated HER2-negative advanced breast cancer. Breast Cancer Res Treat. 2010; 121(1):121-131.

72. Crown J, Dieras V, Staroslawska E, et al. Phase III trial of sunitinib (SU) in combination with capecitabine (C) versus $\mathrm{C}$ in previously treated advanced breast cancer (ABC). J Clin Oncol. 2010;28(Suppl): abstr 18 .

73. Bergh J, Bondarenko IM, Lichinitser MR, et al. First-line treatment of advanced breast cancer with sunitinib in combination with docetaxel versus docetaxel alone: results of a prospective, randomized phase III study. J Clin Oncol. 2012;30(9):921-929.

74. Miller KD, Trigo JM, Wheeler C, et al. A multicenter phase II trial of ZD6474, a vascular endothelial growth factor receptor-2 and epidermal growth factor receptor tyrosine kinase inhibitor, in patients with previously treated metastatic breast cancer. Clin Cancer Res. 2005;11(9):3369-3376.

75. Boér K, Láng I, Llombart-Cussac A, et al. Vandetanib with docetaxel as second-line treatment for advanced breast cancer: a double-blind, placebo-controlled, randomized phase II study. Invest New Drugs. 2012;30(2):681-687.

76. Wood JM, Bold G, Buchdunger E, et al. PTK787/ZK 222584, a novel and potent inhibitor of vascular endothelial growth factor receptor tyrosine kinases, impairs vascular endothelial growth factor-induced responses and tumor growth after oral administration. Cancer Res. 2000;60(8):2178-2189.

77. US National Institutes of Health. PTK787 + Trastuzumab for HER2 Overexpressing Metastatic Breast Cancer. Available from: http:// clinicaltrials.gov/ct2/show/results/NCT00216047. Accessed June 23rd, 2013. 


\section{Publish your work in this journal}

Breast Cancer: Targets and Therapy is an international, peer- View the full aims and scopes of this journal here. The manuscript reviewed open access journal focusing on breast cancer research, identification of therapeutic targets and the optimal use of preventative and integrated treatment interventions to achieve improved outcomes, enhanced survival and quality of life for the cancer patient. management system is completely online and includes a very quick and fair peer-review system, which is all easy to use. Visit http:// www.dovepress.com/testimonials.php to read real quotes from published authors.

Submit your manuscript here: http://www.dovepress.com/breast-cancer---targets-and-therapy-journal 